\title{
LIFETIME AND TAIL SIMULATIONS FOR BEAM-BEAM EFFECTS IN PEP-II B FACTORY*
}

\author{
D. N. Shatilov and A. A. Zholents ${ }^{b}$ \\ a) Budker Institute of Nuclear Physics, 630090 Novosibirsk 90, Russia \\ b) Center for Beam Physics, Accelerator and Fusion Research Division, Lawrence Berkeley \\ Laboratory, University of California, Berkeley, CA 94720
}

\begin{abstract}
A fast tracking technique for doing beam tail simulations has been applied to a study of beam-beam effects in the SLAC/LBL/LLNL PEP-II B Factory. In particular, the dependence of beam lifetime and particle density distribution due to vacuum pressure, damping times, machine nonlinearity and parasitic crossings has been analyzed. Effects of accidental orbit separation and dispersion function at the interaction point (IP) have also been considered.
\end{abstract}

\section{BEAM PARAMETERS AND MODEL}

Beam and machine parameters for PEP-II B factory are described elsewhere [1]. For the sake of completeness, we reproduce in the Table I all parameters we need for a discussion of beam-beam effects. Our notation for most of the parameters has a standard and obvious meaning. Only a few definitions need explanation. In the PEP-II B factory, electron and positron bunches collide head-on at the IP. After the IP, beam orbits are magnetically separated in the horizontal plane. However, before entering its own vacuum pipe, each electron bunch and each positron bunch experiences four more interactions with other bunches of the opposite beam. We refer to these interactions as parasitic crossings (PC's). A parameter $d_{s e p}$ defines orbit separation at the first PC. Orbit separation at the remaining PCs is much larger and, consequently, the effect of beam-beam interactions at these $\mathrm{PC}$ is negligible. We will ignore them in our model and will consider only the first parasitic crossing on either side of the IP. Parameters $\Delta \nu_{x}$ and $\Delta \nu_{y}$ define horizontal and vertical betatron phase advance, in units of the betatron tune, from the main IP to the first PC.

A goal of our study was understanding the mechanisms leading to a beam lifetime limitation in electron-positron colliders. According to experimental observations [2], these mechanisms are fairly insensitive to particle density distribution in the beam core. Thus, a weak-strong model of beam-beam effects seems adequate to our task.

All our simulations were carried out with the beam-beam program LIFETRAC [3]. This program allows the following physics to be included in the simulation:

1. Beam-beam kick.

2. One turn, six-dimensional linear map.

3. Chromaticity up to the third order:

$$
\nu_{x}=\nu_{0 x}+C_{x} \delta+C_{x x} \delta^{2}+C_{x x x} \delta^{3}
$$

\footnotetext{
*Work supported by DOE under Contract DE-AC03-76SF00098 and by Budker INP of the Russian Academy of Science.
}

Table I

Beam parameters

\begin{tabular}{|c|c|c|}
\hline & weak beam $\left(e^{+}\right)$ & strong beam $\left(e^{-}\right)$ \\
\hline$E[\mathrm{GeV}]$ & 3.1 & $\left.9.0^{a}\right)$ \\
\hline$\nu_{0 x}, \nu_{0 y}$ & $34.57,35.64$ & $34.57,35.64^{a)}$ \\
\hline$\nu_{s}$ & 0.037 & $0.052^{a)}$ \\
\hline$\xi_{0 x}, \xi_{0 y}$ & $0.03,0.03$ & $0.03,0.03^{a)}$ \\
\hline$\tau_{x, y}$ [turns] & 7200,7200 & $5014,5014^{a)}$ \\
\hline$\tau_{z}[$ turns $]$ & 3600 & $2507^{a)}$ \\
\hline$\sigma_{\Delta p / p}$ & $0.80 \times 10^{-3}$ & $\left.0.62 \times 10^{-3 a}\right)$ \\
\hline$\sigma_{z}[\mathrm{~cm}]$ & 1.0 & 1.0 \\
\hline$\varepsilon_{x}[\mathrm{~m} \times \mathrm{rad}]$ & $6.4 \times 10^{-8}$ & $\left.4.8 \times 10^{-8} a\right)$ \\
\hline$\varepsilon_{y}[\mathrm{~m} \times \mathrm{rad}]$ & $1.9 \times 10^{-9}$ & $\left.1.4 \times 10^{-9} a\right)$ \\
\hline & \multicolumn{2}{|c|}{ Main crossing } \\
\hline$\beta_{x, y}^{*}[\mathrm{~m}]$ & $0.50,0.015$ & $0.667,0.02$ \\
\hline$D_{x, y}^{*}[\mathrm{~m}]$ & 0,0 & $0,0^{a)}$ \\
\hline$\sigma_{0 x}^{*}, \sigma_{0 y}^{*}[\mu \mathrm{m}]$ & $177,5.3^{a)}$ & $177,5.3$ \\
\hline$d_{\text {sep }}[\mathrm{mm}]$ & \multicolumn{2}{|c|}{ Parasitic crossing } \\
\hline$\sigma_{x}, \sigma_{y}[\mu \mathrm{m}]$ & $284,223^{a)}$ & 243,167 \\
\hline$\Delta \nu_{x}, \Delta \nu_{y}$ & $0.143,0.246$ & $\left.0.117,0.245^{a}\right)$ \\
\hline
\end{tabular}

Here $\delta=\Delta p / p$ and $C_{x}, C_{x x}, C_{x x x}, C_{y}, C_{y y}, C_{y y y}$ are parameters of chromaticity.

4. Machine nonlinearity in the form of an amplitudedependent betatron tune:

$$
\begin{aligned}
& \nu_{x}=\nu_{0 x}+\varepsilon_{x} a_{x x} A_{x}^{2}+\varepsilon_{y} a_{x y} A_{y}^{2} \\
& \nu_{y}=\nu_{0 y}+\varepsilon_{x} a_{x y} A_{x}^{2}+\varepsilon_{y} a_{y y} A_{y}^{2} .
\end{aligned}
$$

Here $A_{x}$ and $A_{y}$ are normalized amplitudes and $a_{x x}, a_{x y}$ and $a_{y y}$ are coefficients.

5. Elastic scattering on nuclei of the residual gas.

6. Parasitic crossings.

7. Dispersion functions at the IP and at the PC.

8. Slicing of a bunch with an arbitrary number of slices (typically, we use 5 pancake-like slices).

9. Orbit separation at the IP.

\section{SIMULATION TECHNIQUE}

The fast tracking technique developed in LIFETRAC [3] emerged from a concept proposed earlier in [4] and realized 
later in [5]. It allows a determination of beam lifetime on a level of 10 hours with a statistical confidence of a few percent by tracking only about $10^{7}$ particle-turns. Along with the lifetime, this technique is able to provide information on the particle density distribution in the beam tails.

The idea of the algorithm is based on the presence of a random component (such as quantum fluctuation noise) in the particle motion [4]. It turns out that a particle's trajectory in phase space depends only on current coordinates and momenta (and noise). A history of the particle's motion is irrelevant for its future trajectory. After accumulating rich statistics of a particle's motion in a certain region of phase space, one can ignore the exact knowledge of a particle trajectory in this region and replace it by statistical information. This information could contain particle coordinates and momenta recorded at the moment when the particle leaves the region. Then, each time the particle's trajectory goes inside that region, one can interrupt tracking and begin a new trajectory from one of the prerecorded points. By this technique, we force actual tracking to go on only in a region with poor statistics rather than tediously tracking in a region with well-defined statistics.

This algorithm was recently checked against 'brute-force' calculations performed with the program TRS [6] and we found excellent agreement in the results [7]. It is worth mentioning that the 'brute-force' calculations took 818 minutes of CPU time on a Cray-2S, while LIFETRAC reached the same accuracy in the tail distribution in 55 minutes of CPU time on a VAX-6610.

\section{RESULTS}

A result of the simulation of beam-beam effects in PEPII for the nominal conditions without PC's is presented in Figure 1a. This plot (and other similar plots) shows particle distribution contours in amplitude space. The first contour corresponds to a particle density a factor $\sqrt{e}$ below the peak and all the following contours correspond to successive reduction with a factor of $e$. Amplitudes $A_{x}, A_{y}$ are normalized amplitudes, i.e. $A_{x}=1$ corresponds to a physical amplitude of $1 \sigma_{0 x}$ and $A_{y}=1$ corresponds to a physical amplitude of $1 \sigma_{0 y}$.

The particle density distribution is obviously perturbed by nonlinear resonances $\ell \nu_{x}+m \nu_{y}+n \nu_{s}=k$. The identified resonances are shown by arrows. On top of each arrow we draw numbers, which correspond to the $\ell, m, n$ resonance identification. Particularly strong is the resonance $14 \nu_{x}=k$. It is partly overlapped with some other resonances, which we were not able to identify.

For the lifetime determination we assumed a limiting aperture of $A_{x}=10$ and $A_{y}=25$. With that aperture we were not able to determine the lifetime, because it was very long. We interrupted calculations when the lifetime exceeded 8 years. We also did not find any blow-up of the beam core.

\section{A. Parasitic Crossings}

Adding PC's did not affect beam lifetime. It was still very long to be determined. But PC's did affect the beam
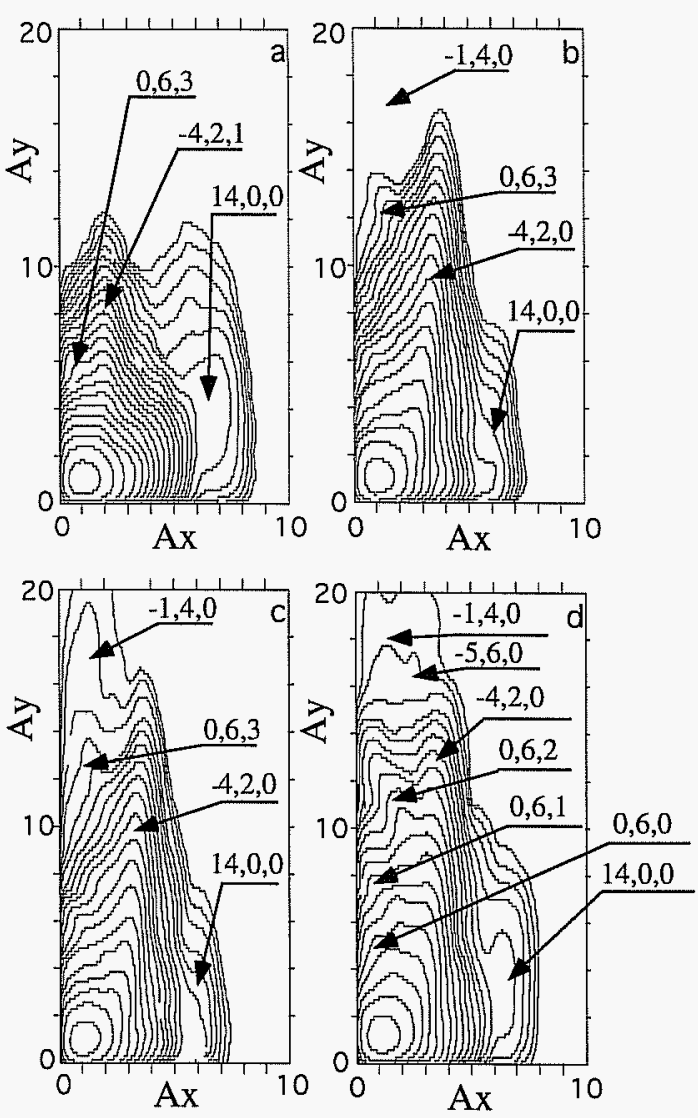

Figure. 1. Particle distribution contours: a) nominal case without parasitic crossings; b) the same as a) plus PC; c) the same as b) plus elastic scattering; d) the same as c), but with $\xi_{0 x}=\xi_{0 y}=0.05$ and $a_{x x}=-200 \mathrm{~m}^{-1}$.

core (we found a $26 \%$ increase in the vertical beam size) and particle density distribution (see Figure 1b). The main factors giving rise to the effect of the PC's are strong resonances: $6 \nu_{y}+3 \nu_{s}=k$ and $-4 \nu_{x}+2 \nu_{y}=k$. At the same time, we found that resonances $-4 \nu_{x}+2 \nu_{y}+\nu_{s}=k$ and $14 \nu_{x}=k$ became weaker. This resonance restructuring is a result of a new beam footprint in tune space in the case with PC's.

\section{B. Vacuum}

Aside from beam-beam effects, the leading mechanism defining the beam lifetime in PEP-II on a level of 23 hours is elastic scattering on nuclei of the residual gas [1]. Since the beam-beam lifetime defined above is much larger, one might think that the beam-beam interaction will have no noticeable effect on the beam lifetime, but this is not right. The interference of beam-beam effects and elastic scattering could be significant. Imagine that the beam-beam interaction creates some resonance islands in phase space close to the aperture limit. Then, particles scattered inside these islands from the beam core, could be trapped there. As a result, the growing population of particles in the tails will decrease beam lifetime. This is exactly what we found when we included elastic scattering [3] in our sim- 
ulation for PEP-II. The lifetime dropped from 22.9 hours (vacuum lifetime) to 16.7 hours. We attribute this to the elastic scattering into the resonance $-\nu_{x}+4 \nu_{y}=k$, which perturbs the particle density distribution at large vertical amplitudes (compare Figure 1c with Figure 1b).

\section{Damping Time}

We compared beam-beam effects for two damping times. First we used a damping time of 5400 turns as it is in [1]; second we took a damping time of 7200 turns, as proposed in [8]. In Figure 2, we show the dependence of the lifetime versus beam-beam parameters for our two cases. The difference between two cases is less than the statistical error expected in the calculations.

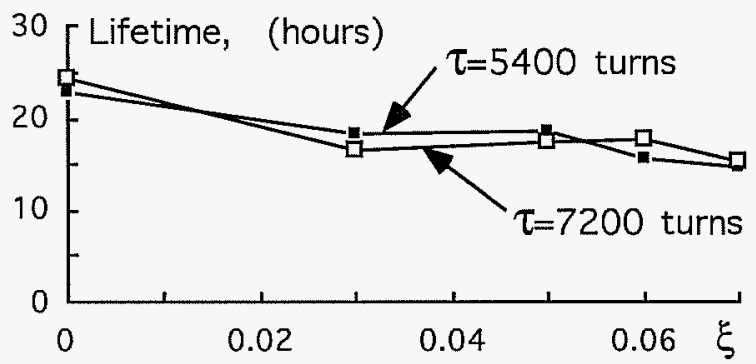

Figure. 2. Beam lifetime versus $\xi=\xi_{0 x}=\xi_{0 y}$ for two damping times. Arrows indicate damping time.

\section{Machine Imperfections}

In order to be more sensitive to the beam-beam effects, we did all the rest of our simulations with $\xi_{0 x}=\xi_{0 y}=0.05$.

Chromaticity. We did not find any significant effect of chromaticity when we varied $C_{x x}, C_{y y}$ in the range of \pm 500 and $C_{x x x}, C_{y y y}$ in the range of $\pm 10^{4}$, which are larger values than we anticipate for the machine.

Tune shifts with amplitude. In our notation, typical dependence of betatron tunes from amplitudes for PEP-II corresponds to $a_{x x}=a_{y y} \sim-200 \mathrm{~m}^{-1}$ and $a_{x y} \sim-1000 \mathrm{~m}^{-1}$ [9]. Simulations with these coefficients gave qualitatively similar results to those with zero nonlinearity. By adjusting $a_{x x}$ with $a_{y y}=a_{x y}=0$ we could slightly increase the strength of the resonance $14 \nu_{x}=k$ at $a_{x x}=-200 \mathrm{~m}^{-1}$ or significantly reduce it at $a_{x x}=400 \mathrm{~m}^{-1}$ (compare Figure 1d and Figure $3 a$ ), but both the beam lifetime and the beam core remained fairly insensitive to this change. For the rest of the simulations we used $a_{x x}=-200 \mathrm{~m}^{-1}$.

Orbit separation at the IP. Table II contain all results. One can see that only the vertical beam size was sensitive to the orbit separation $\Delta x, \Delta y$.

Dispersion at the IP. Simulations with non-zero horizontal and vertical dispersions at the IP showed that the lifetime began to drop below 10 hours when $D_{x}^{*} \geq 4 \mathrm{~cm}$ or $D_{y}^{*} \geq 0.5 \mathrm{~cm}$. An example with $D_{y}^{*}=0.53 \mathrm{~cm}$ is shown in the Figure 3b. Moreover, we found that a dispersion $D_{x}^{*}=2.2 \mathrm{~cm}$ already reduced the lifetime below 10 hours when combined with an accidental orbit separation of $\Delta x / \sigma_{0 x}^{*}=0.4$.
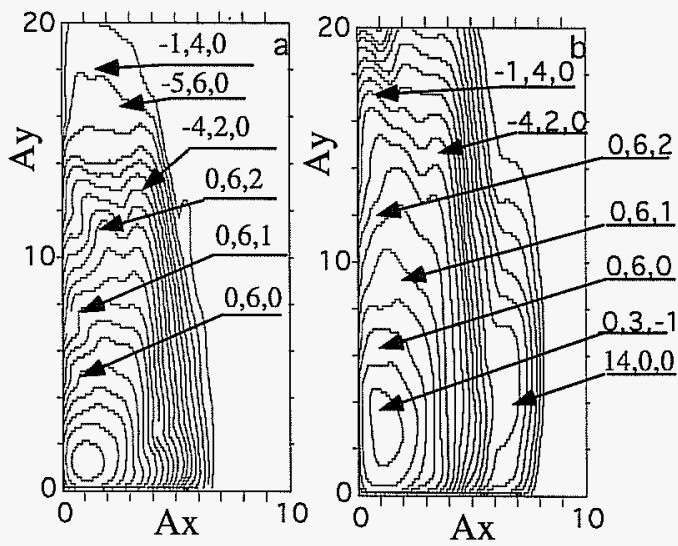

Figure. 3. Particle distribution contours: a) the same as Figure 1d, but with $a_{x x}=400 \mathrm{~m}^{-1}$; b) the same as Figure $1 \mathrm{~d}$ plus $D_{y}^{*}=0.53 \mathrm{~cm}$.

Table II

Orbit separation

\begin{tabular}{|c|c|ccc|}
\hline$\Delta x / \sigma_{0 x}^{*}$ & $\Delta y / \sigma_{0 y}^{*}$ & $\sqrt{\overline{x^{2}}} / \sigma_{0 x}^{*}$ & $\sqrt{\overline{y^{2}}} / \sigma_{0 y}^{*}$ & Lifetime, $[\mathrm{h}]$ \\
\hline 0 & 0 & 1.10 & 1.46 & 18.9 \\
0.2 & 0 & 1.07 & 1.71 & 18.4 \\
0.4 & 0 & 1.12 & 1.93 & 18.4 \\
0 & 0.2 & 1.07 & 1.99 & 19.9 \\
0 & 0.4 & 1.07 & 2.57 & 18.6 \\
\hline
\end{tabular}

\section{Conclusion}

Our study demonstrated that beam-beam effects should not affect the performance of the PEP-II B factory if $\xi_{0 x}=$ $\xi_{0 y}=0.03$. We did not find significant reduction in the beam lifetime even for larger beam-beam parameter, but we did see in many occasions a large increase in the vertical beam size.

Acknowledgment. We are grateful to M. Furman and M. Zisman for many useful discussions.

\section{References}

[1] PEP-II Design Report, SLAC-Report-418, 1993.

[2] A. Temnykh, Third Advance ICFA Beam Dynamics Workshop, INP, Novosibirsk, 1989, p.5.

[3] D.Shatilov, Preprint BINP 92-79, Novosibirsk, 1992, (in russian), unpublished.

[4] J. Irwin, ref.2, p.123.

[5] T. Chen et al., Phys. Rev. E49, 1994, p.2323.

[6] J. Tennyson, unpublished code 'TRS', 1989.

[7] M. Furman et al., CBP Note-59 (1995).

[8] J. Heim et al., Wiggler Insertion of the PEP-II B Factory LER, these proceedings.

[9] E. Forest et al., EPAC 1994, London, 1994, p.1033. 